\title{
LENGUAJE, VERDAD E INTERSUBJETIVIDAD: el influjo de Heidegger en la filosofía de Apel
}

\author{
Language, truth and intersubjectivit: \\ the influence of Heidegger in Apel philosophy
}

\section{Gustavo Salerno}

Doctorando en Filosofía en la Universidad Nacional de Lanús (Argentina). Becario interno del CONICET. Miembro de la Asociación Argentina de Investigaciones Éticas y del Intercambio Cultural Alemán Latinoamericano. Docente en la Facultad de Humanidades de la Universidad Nacional de Mar del Plata. Mar del Plata - Argentina, e-mail: salguz@ gmail.com

\section{Resumen}

El texto pretende una reconstrucción de la recepción que Karl-Otto Apel realiza de algunos aspectos destacados de la filosofía de Heidegger. Ellos aparecen en el contexto de la formación de su idea respecto a la necesidad y posibilidad de una transformación de la filosofía trascendental. Teniendo a la vista este marco, las cuestiones del ser dependiente del lenguaje, la verdad y la intersubjetividad articulan a este trabajo y muestran lo más relevante del influjo heideggeriano en la formación del pensamiento de Apel. En nuestro recorrido seguimos la interpretación de éste, y sólo ocasionalmente presentamos las divergencias de algún investigador reconocido. Hacia el final, incluimos una serie de conclusiones provisorias del estudio propuesto.

Palabras-clave: Lenguaje; Verdad; Intersubjetividad; Conciencia; Transformación. 


\begin{abstract}
It intends a reconstruction of the reception that Karl-Otto Apel does on some Heidegger's philosophy highlights. They appear in context of their idea about the need and possibility of the conscience philosophy transformation. According to this, questions about to be dependant of the language, the truth and the intersubjectivity formulate this work and show the more prominent of Heidegger's influence in the formation of Apel thought. In our way, we will follow his interpretation, and only occasionally will present divergences of some well known investigators. Toward the end, we will include a serie of provisional conclusions.
\end{abstract}

Keywords: Language; Truth; Intersubjectivity; Conscience; Transformation.

\title{
Introduccion
}

En el presente trabajo nos proponemos dar cuenta del influjo que el pensamiento de Heidegger ha ejercido en la filosofía de Karl-Otto Apel. No nos interesa tanto cuestionar la lectura que este último realiza cuanto reconstruir la interpretación que efectivamente ha realizado. Para cumplir con tal objetivo hemos seleccionado tres tópicos en torno a los cuales recorremos las fuentes disponibles: (i) el ser fáctico temporal-finito y su relación con la subjetividad reflexiva y el lenguaje, (ii) el problema de la verdad, y (iii) una incipiente concepción de la intersubjetividad.

El acercamiento de Apel a Heidegger tiene lugar a partir de la tesis doctoral que presenta tomando como eje central a Sein und Zeit. ${ }^{1}$ En realidad se trata, como todo proyecto de investigación, del resultado de una pre-ocupación que constituirá sin embargo el hilo conductor de toda su

1 APEL, Karl-Otto. 1950. Para las citas de la opera maior de Heidegger se utilizará como referencia (salvo que se indique expresamente lo contrario) la traducción de J. E. Rivera Cruchaga. (HEIDEGGER, Martin. Ser y tiempo. Santiago de Chile: Editorial Universitaria. 1997), seguido del número de parágrafo y de página. 
trayectoria filosófica: una primera respuesta a la inquietud de transformar la filosofía trascendental kantiana, tal que superase los atolladeros de la postulación de una cosa en sí incognoscible mediante el análisis de la lingüisticidad en la que estamos inmersos ya siempre que comprendemos. Naturalmente, planteada en estos términos, la problemática tenía que aparecer encaminada a su resolución a través de una gnoseo-antropología que, según Apel, pudiera dar cuenta de lo "irritante" y "fascinante" que se encontraba en Sein und Zeit: "las condiciones ontológicas fundamentales o trascendentales de la comprensión del ser no estaban remitidas al constructo de una «conciencia en general» trascendental, sino a las condiciones «ónticas» de la «facticidad» de «mi propio ser-ahí»-de su «estar arrojado» históricamente y de su «proyecto» conformador del horizonte de sentido" (APEL, 1999, p. 15).

Aparte de la tesis referida, no existe luego un tratamiento especial y exclusivo de Apel para con la filosofía heideggeriana sino que - como resulta característico en su producción - aparecerá abordado en artículos de distinta extensión y temática. En este marco, Apel mismo procede a recapitular, ampliar y modificar su punto de partida inicial conforme su programa de transformación de la filosofía cobra especificidad. En efecto, tienen especial relevancia los trabajos publicados en 1967 (1973), 1989 (1998) y 1991 (1998) ya que muestran cómo, luego de aproximadamente tres décadas, Apel revisa sus ideas originales acerca de la confluencia de dos tradiciones aparentemente opuestas, a saber: la hermenéutica heideggeriana y la filosofía inicial y posterior de Wittgenstein. En la reconstrucción de su pensamiento Apel confirma la plausibilidad de la integración de dichas perspectivas, como así también las dificultades que se sigue de su "historia efectual" (concretamente, en el postmodernismo franco-italiano y en el neopragmatismo norteamericano). En particular Heidegger, si bien junto a otros influjos característicos, será aludido o presentado explícitamente en el reconocimiento de lo que Apel llama a priori de la facticidad.

No podemos dejar de mencionar siquiera sucintamente el hecho de que todas las referencias de Apel a la filosofía heideggeriana - y esto ciertamente a lo largo de sus obras- versan sobre el texto fundamental de éste de 1927 y sobre aquellas que la continúan (concretamente, en el contexto de una crítica del sentido y del alcance de su posterior Kehre). De hecho, a excepción de brevísimas menciones, como por ejemplo una nota al pié en el primer tomo de Transformation der Philosophie del libro de O. Pöggeler Der Denkweg Martin Heideggers en la que se alude al período de las lecciones impartidas entre 1919 y 1923, Apel parece no haberse ocupado del proyecto 
inicial o de la génesis de Sein und Zeit. ${ }^{2}$ La alusión a esta circunstancia que ahora destacamos se justifica en el hecho de que a lo largo de las dos últimas décadas se han venido publicando los cursos y seminarios del período de Freiburg y Marburg en el que Heidegger presenta una serie de indicaciones metodológicas y conceptuales que luego se hayan presupuestas o, al menos, poco desarrolladas en su obra fundamental. Un estudio crítico de la lectura apeliana de Heidegger que comience por incluir en su investigación el material de la fase referida (como, sin embargo, no nos resulta posible realizar aquí) se mostraría sumamente enriquecedor para el programa de una transformación de la filosofía de la conciencia solitaria, proyecto este en el que confluyen los intereses tanto de Heidegger como de Apel.

\section{Facticidad y lenguaje}

Heidegger parte en Sein und Zeit del factum de que nuestra relación con el mundo se encuentra ya siempre mediada simbólicamente, y ello significa: que estamos inmersos en una cierta (pre)comprensión acerca de aquello por lo que preguntamos (el ser). De este modo, Heidegger puede justificar la pretensión de universalidad de su análisis ya que estará dirigida a la explicitación de la "preestructura" (Vorstruktur) de todo comprender. El proyecto filosófico que así se perfila contiene una crítica radical a la tradición metafísica y también -lo cual nos interesa especialmente aquí- al esquema eminente de la moderna filosofía de la conciencia: la relación sujeto-objeto. Como se irá mostrando, la tarea consiste en una transformación hermenéutica de la fenomenología que sustituye pero, a la vez, se orienta en el planteo de una filosofía trascendental. El esquema sujeto-objeto característico del hacer metafísico, filosófico y científico comporta para Heidegger un sentido derivado respecto a una "posición" o "actitud" natural comprensora. Esta modalidad tiene lugar en el Dasein, el cual ha de entenderse metodológicamente como el hilo conductor de la analítica hermenéutica y como un estar-en-el-mundo: en un mundo circundante, propio y compartido. En efecto, "hay un hecho, la comprensión de 'ser' que forma parte esencial de esa realidad que es el ser humano. Investigar

2 La mención que indicamos se encuentra en APEL, 1985, t. I, p. 271 n. 17 acerca del libro de PÖGGELER, 1963, cap. II. Años más tarde Apel reconocerá que su interpretación de la obra heideggeriana- en clave gnoseo-antropológica- se debía al «principio de la significatividad» que E. Rothacker (1966, § 13) contraponía al "principio de la conciencia” de K. Reinhold. Véase APEL, 1998, p. 513. 
éste es lo mismo que ahondar en aquélla. El análisis de la existencia humana como 'lugar' del ser es, pues, el primer estadio de la investigación. Dicho análisis no es la respuesta a cuestión del ser, sino su `elaboración concretá ... su 'horizonte trascendental'." 3

Apel realiza su estudio doctoral en perspectiva kantiana al desatacar los aspectos trascendentales de la "ontología fundamental". Esta preocupación de trasfondo por la indagación de las condiciones de posibilidad y de validez del sentido sirve para interpretar que en Heidegger "la diferencia ontológica, básica para toda su filosofía, entre ser y ente se determina en esa orientación como la distinción kantiana entre los hechos accesibles empíricamente y las formas aprióricas de la percepción" (APEL, 1950, p. 10; APEL, 1985, t. I, p. 229). Las categorías en Kant-siempre según Apel- están referidas a enunciados lógicos, en tanto que para Heidegger no son sino un aspecto parcial del logos que comprende al ser, cito en el ahí $(D a)$ del hombre abierto en la ec-sistencia. El Dasein es en-el-mundo, con lo que una conciencia aislada constituyente del sentido (Husserl) aparece como un modo teorético y objetivante de la filosofía que hay que superar. ${ }^{4}$ Frente al "yo pienso" de la reflexión se impone el más originario "yo soy".

En la analítica existenciaria se trata sobre todo de la elaboración de una pregunta bajo el signo de la transparencia (Durchsichtigkeit), lo cual impone detenerse en el hecho mismo del significado del preguntar. Éste se explicita como la condición en la que se encuentra todo pensamiento referido al mundo y supone una cierta comprensión previa (Vorverständnis) de aquello que se investiga. El preguntar es para Heidegger el comportamiento de un "ente" en particular, el Dasein, que tiene que hacerse su vida, ${ }^{5}$ una vida fáctica no solitaria sino en trato constante con el mundo. De allí que a Apel interese este compromiso mundano peculiar si es comparado con la filosofía precedente,

RODRIGUEZ, 2006, p. 48. Esta caracterización parcial y sumaria simplemente intenta ser una contextualización básica y elemental que resulta necesaria para dar curso al planteo que aquí interesa: el influjo que tiene el proyecto heideggeriano en el camino de la formación del pensamiento de Apel.

4 Para Husserl "la subjetividad es la manera de ser de un ente particular, al que tenemos que llamar sujeto, no en oposición y relación con objetos (Objekte), sino atendiendo a la organización de sus capacidades. Esta lo inviste de su complexión (Verfassung) correspondiente, y lo capacita para conocer, actuar y crear, en suma, para tener un mundo, que él construye según sus propios esquemas" (SZILASI, 1973, p. 28-29; el subrayado es nuestro).

5 HEIDEGGER, 1999, § 4, p. 35: "El Dasein no es tan sólo un ente que se presenta entre otros entes. Lo que lo caracteriza ónticamente es que a este ente le va en su ser este mismo ser." 
toda vez que ahora "el filósofo que reflexiona sobre su pensamiento no es en serio ya el Dios que ha de venir hacia sí, mucho más necesita del autoencasillamiento del pensar finito con contenido" (APEL, 1950, p. 39). Esta fascinación inicial por la "apertura" del ser se prolongará hasta Transformation der Philosophie, principalmente hasta los artículos incluidos en la primera sección de la primera parte, con lo que - como el autor advierte - la obra ha de entenderse también como una transformación de su propia filosofía.

En su escrito doctoral resultaba claro a Apel que la inclusión de la temporalidad en el seno del pensamiento trascendental comportaba un logro que en adelante había que considerar en todo planteamiento de este tipo. La articulación de los tres éxtasis (advenir, sido y presentar) son el Dasein mismo en su autocomprensión como ser finito ante el irrebasable poder-ser en su máxima posibilidad, la muerte. A la afirmación rotunda de que "la muerte es la posibilidad más propia del Dasein. El estar vuelto hacia esa posibilidad le abre al Dasein su más propio poder-ser, en el que su ser está puesto radicalmente en juego" (HEIDEGGER, 1997, § 53, p. 282), Apel agrega: “el `estar vuelto a la muerte' es una no eliminable condición trascendental de posibilidad y validez de la comprensión humana del sentido y con ello también aún del conocimiento discursivo apofántico de objetos" (APEL, 1950, p. 163). ${ }^{6}$

La derivación fundamental de la valoración y de la integración de la temporalidad a las estructuras trascendentales del entendimiento (téngase presente que ya la "tradición" Hamann-Herder-Humboldt cuestionaba el carácter "puro" de la razón kantiana) es la de que representa la forma originaria del comprender, con lo que toda categoría o principio de la razón deben concebirse a priori en el horizonte (Woraufhin) del tiempo (APEL, 1950, p. 174). Esta ponderación de la inserción en el mundo frente a la instancia reflexiva de la racionalidad será revisada más tarde por el propio Apel hasta que su sentido quede casi totalmente transpuesto o, más exactamente, hasta que se muestre la dependencia de todo pensar en y desde la facticidad respecto a una reflexión estricta sobre las presupuestos ineliminables de la argumentación racional.

Si el ser fáctico temporal-finito tiene consecuencias frente a la absolutización de la subjetividad reflexiva, también sucede ello respecto al entrelazamiento de la realidad con el lenguaje. Para revisar dicho aspecto puede sernos útil el trabajo analítico acerca de "las dos fases de la fenomenología",

6 Cf. también APEL, 2004, p. 7-14. Según K. Löwith para Heidegger, como también para Rosenzweig, la muerte significa "una afrenta a la filosofía del 'puro yo' y de la conciencia que no sabe nada sobre este fin empírico del Dasein." (2006, p. 98). 
paradigmáticamente representadas por Husserl y Heidegger. En torno a la concepción del lenguaje en el primero de los filósofos mencionados emerge según Apel una restricción de toda consideración del tiempo y de la historia en el problema de la significación. En efecto, "Husserl distingue las puras "significaciones ideales", cual estrellas fijas en un firmamento supratemporal... de las significaciones realizadas de manera contingente en el fluir de las lenguas históricas. Estas últimas, es decir, los contenidos lingüísticos, sólo pueden funcionar, como en Platón (Carta VII), por participación (metesis) de las puras significaciones eternas" (APEL, 1985, t. I, p. 84). Para Husserl la significación del teorema de Pitágoras permanece temporalmente invariable frente a toda aprehensión individual del mismo. Este enfoque se autocomprende como un intento de superar el peligro del relativismo y del positivismo, quienes reducen el significar y el lenguaje a la posición histórica y a un mero hecho contingente. Sin embargo, tal esfuerzo pierde de vista el cambio evolutivo que se produce en los contenidos significativos, con lo que al pretender salvaguardar la identidad de sentido el filósofo está en condiciones de trascender las lenguas históricas y así "divisar una estructura distinta consistente en ideas, esencias y significaciones puras que sólo posteriormente podrá consolidar en la conciencia con los medios de lenguaje" (APEL, 1985, t. I, p. 85).

La segunda fase de la fenomenología (a la que pertenece en cierto respecto según Apel el último Husserl) aparece caracterizada como el intento de hacer justicia al ser relativo al lenguaje: éste se encuentra aquí entrelazado con el tiempo y con una cultura, sin por ello redundar en relativismo. En efecto, Apel hará privilegiar una tesis que ya no abandonará a lo largo de sus trabajos (aunque irá matizando o condicionando conforme se define su propio programa) que se desprende de la filosofía de Heidegger y que está en clara oposición al Wittgenstein del Tractatus: "es el «lenguaje corriente», no concebible de manera exacta, es decir, puramente objetiva, el que se evidencia como «metalenguaje» último" (APEL, 1985, t. I, p. 89, n. 9). ${ }^{7}$ Se trata de que para Heidegger el ser no es ya un reino de ideas o esencias extramundanas sino el "mundo" abierto como horizonte temporal, permitiendo así en interpretación de Apel reconstruir la diferencia kantiana entre a priori y a posteriori como diferencia ontológica entre ser y ente. Esta diferenciación no es, sin embargo, un dualismo redivivo sino que se presenta como una articulación "dialéctica": "sin el hombre existente fácticamente, el ser no puede despejarse al mundo; por otro lado, el hombre se

7 Acerca de esta concepción véanse también APEL, 1985, t. I, p. 182 y ss; APEL, 1989, p. 70; APEL, 1994, p. 195-196. 
encuentra ya él mismo en el claro de la comprensión del ser" (APEL, 1985, t. I, p. 91). En el primer momento se hace presente el punto de vista óntico -o, como Apel mismo entiende, la afección sensible kantiana -, y en el segundo la comprensión del ser en un contenido sistemático: el lenguaje.

Por tanto, este influjo heideggeriano lleva a la profundización en una concepción trascendental del lenguaje que luego Apel irá ampliando, especialmente a partir de la integración en su programa de la filosofía analítica y de la hermenéutica de Gadamer. Por el momento es menester aquí volver sobre la mencionada "fascinación" de Apel al inicio de sus trabajos por la temporalización del lenguaje, toda vez que representa en la analítica del ser un aspecto fundamental. Para Heidegger debía hablarse de una "fundación lingüística del ser", expresión que alude a lo constitutivo de la comprensión que opera en toda percepción auténtica, correspondiente a la imaginación trascendental de Kant. Así, en autorreferencia a su desarrollo intelectual, Apel afirma: "en mi escrito de habilitación... desarrollé - inspirado por Heidegger una posición filosófica fundamental muy semejante a la que más tarde encontré en el opus magnum de Gadamer. La denominé "hermenéutica trascendental", para dejar constancia de mi adhesión al planteamiento kantiano. Pues en aquel entonces - de manera semejante a Gadamer con su concepción de una `hermenéutica filosófica - me parecía posible entender la hermenéutica del Dasein como una transformación necesaria y suficiente de la filosofía trascendental de Kant y de Husserl, en el sentido de una atención a la lingüisticidad (Sprachlichkeit) y la historicidad (Geschichtlichkeit) de nuestro 'estar-en-el-mundo' (In-der-Welt-seins)" (APEL, 1998, p. 571). ${ }^{8}$

En otro lugar Apel amplía la distinción entre los proyectos fenomenológico husserliano y hermenéutico heideggeriano en torno al aspecto que venimos tratando. Frente al carácter eminentemente descriptivo de la conciencia trascendental tanto de la realidad como de los otros (Husserl), la transformación hermenéutica de la fenomenología llevada a cabo por Heidegger parte de una precomprensión e interpretación pública del Dasein, con lo que el método consistente en pensar con el lenguaje y en dependencia del lenguaje como medium histórico se muestra como el logos característico de la filosofía heideggeriana. De esta forma Heidegger supera el enfoque eidético orientado antepredicativamente que es peculiar en Husserl en dirección a una hermenéutica del estar-en-el-mundo ya siempre interpretado lingüísticamente. Apel mismo

8 La tesis de habilitación de Apel referida en la cita lleva por título Die Idee der Sprache in der Tradition des Humanismus von Dante bis Vico (BONN, 1963). 
recuerda el pasaje en el que Heidegger se refiere a la imposibilidad del Dasein de trascender el estado de interpretado en el que se encuentra ya siempre y respecto del cual tiene lugar todo comprender, interpretar y comunicar. En efecto, aquí reside la "clave de una hermenéutica del lenguaje", con lo que "en Sein und Zeit se pone ya en juego una dimensión de la "temporalidad" o "historicidad" de todo comprender que en Wittgenstein no está articulada" (APEL, 1998b, p. 469). ${ }^{9}$

\section{El problema de la verdad}

Hasta aquí, como puede observarse, hemos avanzado en el repaso de ciertas coincidencias de Apel con la filosofía heideggeriana -y esto no sólo en relación con sus primeros trabajos-. Sin embargo, la influencia y sus implicancias de la "apertura del ser" pronto cederán lugar a un posicionamiento crítico respecto de Heidegger, lo cual sucede sobre todo a partir de la interpretación apeliana del concepto de verdad de aquel. En efecto, junto con la recepción de la obra fundamental de Gadamer, la cuestión mencionada se yergue como la clave del alejamiento apeliano de la Erschlossenheit del mundo del que depende todo sentido, y a través de la cual -precisamente - acontece la verdad.

Para Heidegger la analítica existencial explicitaba que las cosas intramundanas comparecían en las modalidades de lo Vorhandenheit y lo Zuhandenheit en virtud de la apertura previa del mundo, lo cual se mostraba además como la condición de posibilidad de la emergencia y del ser de tales entes. Sin embargo, también resultaba claro que el ser del mundo y del Dasein que es en él era radicalmente distinto, y ello porque el Dasein carece de toda condición respectiva: “que el Dasein está 'iluminado' ('erleuchtet') significa que, en cuanto estar-en-elmundo, él está aclarado en sí mismo, y lo está no en virtud de otro ente, sino porque él mismo es la claridad (Lichtung)" (HEIDEGGER, 1997, § 28, p. 157). Tal abrirse hacia sí es sin dudas una posibilidad característica del Dasein, y desde ella enfrentará al problema de la verdad: más precisamente, criticará el concepto tradicional de la verdad como correspondencia (adaequatio) y sus fundamentos ontológicos,

$9 \quad$ El pasaje de Sein und Zeit al que se alude es el siguiente: "El Dasein no logra liberarse jamás de este estado interpretativo cotidiano en el que primariamente ha crecido. En él, desde él y contra él se lleva a cabo toda genuina comprensión, interpretación y comunicación, todo redescubrimiento y toda reapropiación. No hay nunca un Dasein que, intocado e incontaminado por este estado interpretativo, quede puesto frente a la tierra virgen de un 'mundo' en sí, para solamente contemplar lo que le sale al paso” (HEIDEGGER, 1997, § 35, p. 192). Cf. también acerca de las diferencias con el proyecto husserliano APEL, 1985, t. I, p. 266 y ss. 
mostrando el carácter derivado del mismo. En efecto, si se comprende correctamente a Aristóteles, para Heidegger hay que concluir: "ser-verdadero (verdad) quiere decir ser-descubridor [...] El ser-verdadero del logos en cuanto apofansis es el alhtenein en el modo del apofainestai: un hacer ver al ente en su desocultación (en su estar al descubierto), sacándolo fuera del ocultamiento" (HEIDEGGER, 1997, § 44, p. 239). Por tanto, de acuerdo a Sein und Zeit, las cosas intramundanas son en tanto que están descubiertas (Entdecktheit) y dependen de la actividad descubridora, es decir: tienen como condición de posibilidad la originaria aperturidad del Dasein (Erschlossenheit des Dasein). De allí la conclusión: "en tanto que el Dasein es esencialmente su aperturidad, y que, por estar abierto, abre y descubre, es también esencialmente 'verdadero'. El Dasein es 'en la verdad'. Este enunciado tiene un sentido ontológico" (HEIDEGGER, 1997, § 44, p. 241). ${ }^{10}$

La posición de Apel frente a esta concepción recoge las observaciones críticas que realizara E. Tugendhat en su estudio sobre el concepto de verdad en Husserl y Heidegger. ${ }^{11}$ Básicamente, Apel destaca el problema de la justificabilidad o de los cánones de enjuciamiento de la teoría (de la verdad) que pretende validez universal. Ello ocurre en la medida en que Heidegger identifica la apertura del sentido con la verdad en la modalidad de la «aletheia» originaria. Pero esto significa en el contexto heideggeriano que la apertura (Eröffnung) es, a la vez, ocultación del sentido, con lo que posibilita sólo bajo determinadas circunstancias la verdad de los enunciados. Ahora bien, a pesar de esta dependencia, únicamente en este último caso es posible encontrar una instancia objetiva (el ente, lo enunciado) con la cual comprobar o justificar lo que ha sido afirmado, pero no en el caso de la apertura despejadora-ocultadora. Aquí falta la diferencia sujeto-objeto, de modo que "si identificamos la verdad con el «despejamiento» (Lichtung) del sentido, puede revelarse como asunto (Sache) del «destino», del que ya no somos responsables" (APEL, 1985, t. I, p. 40). ${ }^{12}$ Tras la Kehre, es decir, luego del retorno de la analítica existencial a un

10 Además: "El Dasein, en cuanto constituido por la aperturidad, está esencialmente en la verdad. La aperturidad es un modo de ser esencial del Dasein. 'Hay'verdad sólo en cuanto y mientras el Dasein es. El ente queda descubierto cuando y patentizado mientras el Dasein es" (HEIDEGGER, 1997, § 44, p. 246; subrayados en el original).

11 TUGENDHAT, 1967.

12 Según Apel, Heidegger ha confirmado - quizá sin conocimiento de la crítica de Tugendhat

- la insuficiencia del concepto de verdad (aletheia) qua despejamiento (Lichtung) en HEIDEGGER, 1969, p. 76 y ss. En virtud de esta rectificación del propio autor, Apel no incluye la teoría de la aletheia en su discusión con los modelos criteriológicamente relevantes de la misma. APEL, 1995, p. 45-46. 
pensar desde la pertenencia a la historia del ser, la problemática de la validez o de la justificabilidad (en definitiva, de la trascendentalidad) quedaba incluida en la tradición metafísica que había que destruir. De allí también que Apel haya destacado siempre la importancia del pensamiento heideggeriano en su aspecto cuasi-trascendental: es decir, sólo en el sentido de su interés por las condiciones de posibilidad de la constitución del sentido, aunque deficitariamente orientado ante y por el problema de la validez. Su esfuerzo consistirá en alcanzar una ampliación o profundización de la hermenéutica heideggeriano-gadameriana en el sentido de una hermenéutica trascendental.

Al hilo de la crítica a la concepción de la verdad como aletheia Apel se distanciará de la "historia efectual" de Heidegger, la cual conduce hacia la detrascendentalización e historización de la filosofía. Aparte del detalle de estas críticas -las cuales por el momento podemos obviar- interesa atender al hecho de que para Apel en esas orientaciones, por así decir, se sacan las conclusiones o implicancias del propio enfoque heideggeriano. Se eleva entonces como problema la posibilidad de una transformación de la filosofía trascendental que, sin embargo, mantenga la exigencia kantiana de la questio iuris. Y es que "para Kant la pregunta (y posible contestación a la pregunta) por las condiciones de posibilidad de la constitución del objeto, es decir, de la constitución del sentido de la objetividad era idéntica a la pregunta (y la posible contestación a la pregunta) por las condiciones de posibilidad de la validez intersubjetiva del conocimiento verdadero." 13

\section{La cuestión de la intersubjetividad}

El tercer aspecto que se destaca en la formación del pensamiento de Apel en su trato con la filosofía heideggeriana se perfila en la dirección de una incipiente concepción de la intersubjetividad. Como se dijo más arriba, para Hedeigger frente al reflexivo "yo pienso" que se aísla y constituye el sentido en virtud de sus evidencias concienciales, el Dasein es en-el-mundo, y esto significa: en un mundo propio (Selbstwelt), en un mundo circundante (Umwelt) y en un mundo compartido con otros (Mitwelt). Todos ellos son modos cooriginarios del ser, y sólo en la descripción hermenéutico-fenomenológica se

13 APEL, 1998b, p. 511. Cf. KANT, 1978, A 111 (p. 193): "Las condiciones a priori de la experiencia en general son, a la vez, condiciones de posibilidad de los objetos de la experiencia." 
hallan destacados por separado. De esta forma, toda comprensión tiene lugar en-cada-caso (je schon) en un mundo articulado con otros, y el filósofo tiene que poder explicitar dicho horizonte de posibilidad del sentido.

La idea de una intersubjetividad lingüísticamente articulada se muestra sobre todo - en un Apel de inspiración heideggeriana - a partir de la discusión que realiza de la que considera la tesis fundamental de la filosofía del lenguaje de Wilhelm von Humboldt. Se trata de que para Humboldt todo pensar se encuentra anclado en un lenguaje que transmite una determinada imagen del mundo que resulta irrebasable. Esta interpretación prerreflexiva del problema de la verdad se adhiere a cualquier intento de comprensión que el individuo pretende alcanzar por medio del lenguaje que emplea. Ello sirve a Apel para argumentar, frente a Ockham y Leibniz, a favor de la imposibilidad de ir por detrás de "la apertura concreta e histórica del sentido que se da en un mundo abierto por el lenguaje y en la cual el ente - incluyendo en su concepto lo que el hombre mismo es - nos hace frente como "algo" dentro de unos "contextos referenciales" (APEL, 1985, t. I, p. 119).

En efecto, una lingüística "orientada al contenido" hace suya la idea de que las estructuras del lenguaje están fundadas en las "visiones" del mundo que ellas articulan. Este enraizamiento del pensar y el comprender en un logos concreto e histórico (y, por tanto, relativo a una "cosmovisión"), sin embargo, no imposibilita para Apel pensar su dependencia respecto a un logos "en general", nuevamente - aunque ahora no utilice esta expresión - en términos "dialécticos". De hecho "el lenguaje es sencillamente el medio único e insustituible en el cual el pensamiento tendente a la validez universal y, en esa medida, "excéntrico", es decir, que desborda toda perspectiva humana ligada a lo corporal $[. .$.$] se integra siempre dentro de las visiones del mundo relativas a$ una perspectiva - otras no puede haber - y, por tanto, ligadas a lo corporal" (APEL, 1985, t. I, p. 126). Por lo tanto, sólo en la verdad abierta en el lenguaje queda asegurada una orientación coherente en el mundo, la cual es compartida en el marco de una comunidad lingüística; es decir: en sentido "material" o "corporal" todo sujeto se inserta dialécticamente en una comunidad de la que depende y, a la vez, trasciende (para ser nuevamente remitido a ella), y que preludia lo que luego se presentará en la pragmática trascendental como comunidad real de comunicación y comunidad ideal de comunicación.

Es en este sentido que para Apel el mundo compartido en el que ya siempre estamos insertos y en el que nos relacionamos cultural e históricamente refiere a un Dasein que no se encuentra separadamente de los otros. Esta concepción puede presentarse como una forma incipiente de 
comprender la intersubjetividad, perspectiva que ab initio de su desarrollo intelectual Apel toma explícitamente de Heidegger: su tesis doctoral (1950), el primer artículo (primera sección, primera parte) de Transformation der Philosophie (1955-1957), y su tesis de habilitación (1963) así lo muestran. Sin embargo, pronto irán agregándose otros influjos que limitarán y hasta reformularán críticamente este punto de arranque.

La atención de Apel a la filosofía del lenguaje de von Humboldt resulta significativa aquí ya que este filósofo ha sido una de las inspiraciones principales en la tradición en la que se prepara y desarrolla la función de "apertura del mundo" (por medio) del lenguaje, a la que ciertamente pertenece también Heidegger. En este orden, para C. Lafont la tríada Hamann-Herder-Humboldt avanza hacia una idea de lenguaje que tiene como punto de partida una crítica a la concepción instrumentalista de aquel, característica de la "filosofía moderna de la conciencia". De ello se siguen por lo pronto dos rasgos significativos del "giro lingüístico" promovido por dichos filósofos: (i) el lenguaje se muestra como la condición de posibilidad de la objetividad de la experiencia y, a la vez, de la comunicación intersubjetiva; (ii) lo cual resulta posible si se asume el costo de una detrascendentalización. En efecto, las lenguas históricas de las que parten Hamann-Herder-Humboldt no pueden asumir el sitio privilegiado de una conciencia en general que ha sido desterrada ya que la pluralidad de aquellas se torna en definitiva inconmensurable, y debido a que tanto la verdad como la referencia quedan pendientes de la posible apertura lingüística del mundo.

El acento humboldtiano (como característica eminente de la tradición de la que se habla) en la idea de que a cada lenguaje le pertenece una concepción del mundo peculiar debe cotejarse aquí, en suma, con la siguiente circunstancia: la de que "de este tipo de giro lingüístico se siguen, en relación con la intersubjetividad de la comunicación[...] las conocidas limitaciones, de corte relativista, respecto a la posibilidad de entenderse sobre lo mismo o, en el mismo sentido, respecto a la posibilidad de traducción de un lenguaje a otro" (LAFONT, 1997, p. 24) ${ }^{14}$ La fuerza de esta tesis dependerá en definitiva de cuál sea el concepto de mundo en el que se piense, es decir: será cierta si y sólo si se relativiza la afirmación de que "para clasificar imágenes del mundo es necesaria la idea explícita de mundo en general. Y si el 'mundo' es un

14 En el análisis de Lafont, Heidegger es interpretado como un "idealista" al hipostaziar el factum de la función de la "apertura del mundo" del lenguaje (es decir, nuestra relación simbólicamente mediada con el mundo). Ello se debe al hacer suya la concepción lingüística de Humboldt que redunda - en Heidegger - en un prejuzgamiento anticipado de todo "hacer frente" intramundano. 
constitutivum del Dasein, la elaboración conceptual del fenómeno del mundo exige una visión de las estructuras fundamentales del Dasein" (HEIDEGGER, 1997, § 11, p. 77). Con otras palabras: frente a la cuestión mundo, en Heidegger debe tenerse en cuenta que "es claro que se trata de un carácter formal, de tal manera que, para los efectos del análisis, es indiferente el mundo particular" (AGUILAR-ÁLVAREZ BAY, 2004, p. 133; el subrayado es nuestro).

Por el momento, más allá de las divergencias en torno a la interpretación correcta del aspecto referido, tendremos que justificar la afirmación vertida más arriba acerca de que la idea heideggeriana de un mundo compartido inspira la problemática de la intersubjetividad en Apel, y lo haremos recordando brevemente el trasfondo del que surge. A partir del capítulo cuarto de Sein und Zeit, Heidegger intenta dar respuesta a la pregunta acerca de quién es en-el-mundo: "la aclaración del estar-en-el-mundo ha mostrado que no 'hay' inmediatamente, ni jamás está dado un mero sujeto sin mundo. Y de igual modo, en definitiva, tampoco se da en forma inmediata un yo aislado sin los otros" (HEIDEGGER, 1997, § 25, p. 141). Para Heidegger, en adelante, la tarea consistirá en explicitar hermenéuticamente el sentido de esta coexistencia (Mitdasein) en la cotidianidad: con ello no se alude a la mera convivencia sino al Dasein de los otros que están conmigo; se trata en todo caso de un co-Dasein (el cual será abordado al hilo del "indicador formal" de la existencia) que realiza efectivamente un mundo en común (Mitwelt).

La estructura ontológica-existencial es entonces un coestar-conlos-otros (Mitsein mit Anderen), con lo que queda preanunciado uno de los modos de hacer frente al solipsismo metodológico de la filosofía moderna, tal como el propio Apel afirma. ${ }^{15}$ Ello enlaza con la crítica heideggeriana a Descartes y a los fundamentos metafísicos de su filosofía. Es decir, se trata del problema del solipsismo inherente a la concepción que sostiene que toda realidad - incluido el ego- es substantia, res o cosa. En este marco, el yo aparece como un receptaculum autárquico de evidencias en la cual se dan las ideas o representaciones de las cosas (y no estas mismas), con las cuales puede alcanzarse un conocimiento indubitable. Quisiéramos destacar que esta es una aporía fundamental de la filosofía precedente que Apel cree imperioso transformar y, sobre todo, explicitar la influencia de Heidegger en la formación de dicha necesidad. En efecto, para éste "Descartes deja sin examinar el sentido

15 por ejemplo, APEL, 1950, p. 61; también (aunque ya en camino hacia una "hermenéutica trascendental"), APEL, 1985, t. I, p. 23-24; t. II, p. 169 y ss; 315 y ss. APEL, 2004, p. $259-260$ 
del ser involucrado en la idea de sustancialidad y el carácter de 'universalidad' de esta significación", con lo que "no sólo elude la pregunta ontológica por la sustancialidad, sino que también afirma explícitamente que la sustancia como tal, es decir, la sustancialidad, es inaccesible en sí misma y por sí misma" (HEIDEGGER, 1997, § 20, p. 120).

\section{Conclusiones provisorias}

Provisionalmente podemos extraer algunas conclusiones del repaso de la recepción apeliana de la filosofía de Heidegger, pendientes de una sistematización y desarrollo más profundos. Éstas son tres. La primera se circunscribe estrictamente al problema planteado en el párrafo anterior y consiste en el reconocimiento de que la tesis (relevante para una crítica del sentido) que expresa que al preguntar por el modo de acceder a la realidad exterior se ha presupuesto ya siempre a ésta resulta posible en virtud de los propios presupuestos básicos de Sein und Zeit. Y es que "la significatividad misma, con la que el Dasein ya siempre está familiarizado, lleva empero consigo la condición ontológica de la posibilidad de que el Dasein comprensor pueda abrir, en cuanto interpretante, algo así como `significaciones', las que por su parte fundan la posibilidad de la palabra y del lenguaje" (HEIDEGGER, 1997, § 18, p. 114). Por ello Apel enfatiza que "el "escándalo" del problema del mundo exterior (como también el del problema de la existencia de otros hombres) no está, según Heidegger, como también según Wittgenstein, en la falta de una prueba suficiente, como pensaba aún Kant, sino en la demanda de una prueba" (APEL, 1985, t. I, p. 250, n. 54). Se trata de que, frente a la exigencia de una demostración de la existencia de las cosas "fuera de mín", hay que notar que "el 'escándalo de la filosofía' no consiste en que esta demostración aún no haya sido hecha, sino más bien, en que tales demostraciones sigan siendo esperadas e intentadas" (HEIDEGGER, 1997, § 43, p. 226. Apel vuelve a destacar este aspecto en 1998, p. 471).

El segundo aspecto a destacar es el de que, si bien Apel considera que Heidegger insiste en que el Dasein está ya siempre con (otros) no por ello logra escapar a cierta forma de solipsismo. Ésta se juega en el hecho de que el criterio último para la validación del sentido y, sobre todo, del discurso filosófico, depende del "por mor de qué" (Worumwillen) del Dasein: la resolución (Erschlossenheit) hacia la propiedad sólo es tal en la medida en que se singulariza, es decir, en tanto puede superarse la "caída" 
en el Uno (das Man). Por tanto, "en la unidad básica de la comprensión qua apertura de $m i$ ser-en-el-mundo, no está suficientemente considerada la tensión entre la «interpretación pública» del mundo, mediada lingüísticamente, y mi experiencia del mundo" (APEL, 1985, t. II, p. 191, n. 50). Mediante la interpretación que K. Löwith brinda de Heidegger también surge que el solipsismo es un peligro que se desprende de la filosofía de éste, si bien el discípulo está preocupado por destacar la transformación que comporta respecto del paradigma precedente: el pensamiento que comienza por reconocer la facticidad del propio estar-ahí niega por principio toda filosofía que tiene su punto de partida en el yo o en la autoconciencia puros, con lo que "el sentido y el ser del 'yo soy' no surge de un yo universal, sino del pronombre personal de primera persona que siempre es sólo en cada caso yo mismo" (LÖWITH, 2006, p. 102). No obstante, Bertorello sostiene que, ceñidos al marco de la obra de 1927, la crítica apeliana es atendible, "pero si se confronta con las obras del período de Marburg donde Heidegger señala permanentemente que el ser del Dasein se da en el medio del lenguaje, la objeción deja de tener sentido, ya que la instancia de la enunciación siempre supone la intersubjetividad o, lo que es lo mismo, el Mitsein. La propiedad de la existencia no suprime la convivencia (Miteinandersein) con los otros, sino que significa modificar la convivencia" (BERTORELLO, 2007, p. 142).

La tercera cuestión de importancia tiene la forma de un balance respecto del alcance que, en definitiva, puede tener la transformación heideggeriana de la filosofía, y de sus consecuencias para la cuestión de la intersubjetividad. Según Apel, el interés fundamental que comparte su programa filosófico con el de Heidegger consiste en la preocupación por encontrar una respuesta a la pregunta por "las condiciones de posibilidad del comprender el mundo [Welt-Verstehen] y, en esa medida, de la constitución del sentido del mundo [Welt-Sinnkonstitution]" (APEL, 1998, p. 510). Ahora bien, cuando se apela a Kant, siempre debe tenerse en cuenta el dictum acerca de que las condiciones a priori de la experiencia en general son, a la vez, condiciones de posibilidad de los objetos de la experiencia, y ello significa para Apel que las preguntas y las respuestas acerca de la constitución del sentido y la validez intersubjetiva del conocimiento verdadero tienen que ser idénticas. Si se tiene presente todo ello, habría que acordar con el análisis apeliano en que $(a)$ Kant en el contexto de una crítica de la razón pura - sólo se ocupó de investigar la forma de la objetividad pero no sus contenidos concretos (lo que, por otro lado, le permitía fundamentar categorías aprióricas, es decir, válidas universal y 
necesariamente); y (b) Heidegger - en el marco de una hermenéutica de la facticidad- no pudo asegurar el nexo validez-constitución del sentido ya que en él "la posible verdad judicativa es siempre ya dependiente del despejamiento y ocultamiento [Verbergung] precedentes del sentido del ente" (APEL, 1998, p. 512). Así considerado, el planteo del problema de la validez intersubjetiva, y la transformación buscada de la filosofía trascendental kantiana, quedaban relativizados.

\section{Referências}

AGUILAR-ÁLVAREZ BAY, T. El lenguaje en el primer Heidegger. México: FCE, 2004.

APEL, K.-O. Dasein und Erkennen. Eine Erkenntnistheoretische Interpretation der Philosophie Martin Heideggers. Bonn: RheinnischenFriedrich-Wilhelm Universität, 1950.

Autopercepción intelectual de un proceso histórico. Anthropos, 183, p. 12-19, marzo/abr. 1999.

La ética del discurso ante el desafío de la filosofía latinoamericana de la liberación (II). In: APEL, Karl-Otto; DUSSEL, Enrique. Ética del discurso y ética de la liberación. Madrid: Trotta, 2004a.

Wittgenstein y Heidegger: la pregunta por el sentido del ser y la sospecha de carencia de sentido dirigida contra toda metafísica. In: Diánoia, México, XIII, 1967; y Philosophisches Jahrbuch, 75, p. 56-94, 1967; recogido luego en APEL, Karl-Otto. La transformación de la filosofía, 1985b, t. I, p. 217-264).

"Sinnkonstitution und Geltungsrechtfertigung. Heidegger und das Problem der Transzendentalphilosophie", In: FORUM FÜR PHILOSOPHIE BAD HOMBURG (Hrgs.). Martin Heidegger: Innen- und Außenansichten. Frankfurt a. M: Suhrkamp. 1989c, p. 131-175; recogido luego In: APEL, KarlOtto. Auseinandersertzungen. 1998b, p. 506-568.

Wittgenstein und Heidegger. Kritische Wierderholung und Ergänzung Eienes Vergleichs. en: McGUINNESS B. Der Löwe Spricht... und Wir Konnen ihn Nicht Verstehen. Frankfurt a. M: Suhrkamp. 1989, p. 131-175; recogido luego In: APEL, Karl-Otto. Auseinandersertzungen. 1998b, p. 459-503. 
¿Es la muerte una condición de posibilidad de la significación? (1976).

Agora, v. 10, p. 7-14, 2004b.

Falibilismo, teoría consensual de la verdad y fundamentación última.

en: ____. Teoría de la verdad y ética del discurso. Barcelona: Paidós, 1995. p. 37-145.

El desafío de la crítica total a la razón y el programa de una teoría filosófica de los tipos de racionalidad. In: Anales de la cátedra Francisco Suárez, v. 29, p. 63-95, 1989.

Semiótica filosófica. Buenos Aires: Almagesto, 1994.

Regulative Ideen oder Wahrheits-Geschehen? Zu Gadamers Versuch, die Frage nach den Bedingungen der Möglichkeit gültigen Verstehens zu beanworten. In: APEL, Karl-Otto. Auseinandersertzungen. 1998b, p. 569-607.

La transformación de la filosofía. Madrid: Taurus, 1985a.2 t.

BERTORELLO, A. La reflexividad del logos hermenéutico. El problema de la universalidad en la filosofía de Sein und Zeit. In: MICHELINI, D.; MALIANDI, R; De ZAN, J. (Ed.). Ética del discurso: recepción y críticas desde américa latina. Río Cuarto: ICALA, 2007. p. 129-142.

ROTHACKER, E. Zur Genealogie des Menschlichen Bewusstseins. Bonn: Bouvier, 1966.

LÖWITH, K. Heidegger, pensador de un tiempo indigente: sobre la posición de la filosofía en el siglo XX. Buenos Aires: FCE, 2006.

KANT, I. Crítica de la razón pura. Madrid: Alfaguara, 1978.

LAFONT, C. Lenguaje y apertura del mundo: el giro lingüístico en la hermenéutica de Heidegger. Madrid: Alianza, 1997.

RODRIGUEZ, R. Heidegger y la crisis de la época moderna. Madrid: Síntesis, 2006.

HEIDEGGER, M. Ser y tiempo. Santiago de Chile: Editorial Universitaria, 1997.

Zur Sache des Denkens: Tübingen, 1969 (GA 14). Tradução de Garrido-Molinuevo-Duque. Tiempo y ser. Tecnos: Madrid, 1999. 
PÖGGELER, O. Der Denkweg Martin Heideggers. Pflullingen: Verlag, 1963.

SZILASI, W. Introducción a la fenomenología de Husserl. Buenos Aires: Amorrortu, 1973.

TUGENDHAT, E. Der Wahrheitbegriff bei Husserl und Heidegger. Berlin: W. de Gruyter, 1967.

Recebido: 05/05/2008

Received: 05/05/2008

Aprovado: 25/05/2008

Approved: 05/25/2008 